\title{
THE STUDY OF OSCILLATIONS LOCALIZED NEAR A LAYER OF DENSITY DISCONTINUITY IN AN EXAMPLE OF SOLAR MODEL
}

\author{
Yu. V. Vandakurov \\ A.F. Ioffe Physical Technical Institute \\ 194021, Leningrad \\ USSR
}

If at some radius $r=r_{j}$ there is a jump of the density and composition then a simple asymptotic formula can be derived which gives $\omega$, the oscillation frequency for large degree modes $(\ell \gg 1)$. Using relations of Gabriel and Scuflaire (1979) and taking into account the fact that the oscillation amplitude at $R\left\langle r_{j}\right.$ or $R>r_{j}$ is equal to $r^{\ell}$ or $r^{-\ell}$ multiplied by some functions only weakly dependent on $\ell$, we obtain

$$
\omega^{2}=\ell\left\{\frac{g\left(\rho_{i}-\rho_{e}\right)}{r\left(\rho_{i}+\rho_{e}\right)}\right\}_{r-r_{j}},
$$

where $g$ is the gravitational acceleration, and $\rho_{i}\left(\rho_{e}\right)$ are the densities at the lover (upper) sides of the layer in question. The comparison with the exact $\omega$-values considered below shows that the asymptotic values are larger by 13 , 8.8, and 6 percents for $\ell$ equal to 5,7 , and 10 , respectively. Because $\omega$ should be less than the maximum value of the Brunt - Văisălä frequency, we have for a finite thickness $\Delta$ of the jump layer and for finite $\ell$ that $\ell \ll 2 r_{j} / \Delta$. We have performed numerical study of the above modes which may be called interior modes. The equilibrium model was similar to that studied by Vandakurov (1984 a,b) but now we assume that a small iron-like core (as in Rouse's (1983) model) is present. We assume the hydrogen content in the core to be large enough for convection to occur. Above the core, there are the inhomogeneous convectively neutral zone $\left(r_{j} \leq r \leq r_{11}\right)$, the homogeneous radiative zone $\left(r_{j 1} \leq r \leq r_{u}\right)$, and the convective envelope $\left(r_{\mathrm{u}} \leq r \leq R\right)$, whose structure have been taken from Spruit's (1974) paper.

The parameters of the model which has been calculated were not found to be appropriate enough to reproduce solar characteristics. For instance, to get the right luminosity, the energy generation due to $p-p$ reaction should be approximately 3 times larger than that following from the common formula. Therefore we shall not describe the model in detail, and only mention that the relative values of radius and mass of the core are equal to 0.047 and 0.0092 , respectively, and $\rho_{\mathrm{i}} / \rho_{\mathrm{e}}=2.6$. This jump in the density is due to the change in the hydrogen and heavy elements abundances for which $X_{i}=0.035$, $Z_{\mathrm{i}}=0.90, X_{\mathrm{e}}=0.63$, and $Z_{\mathrm{e}}=0.2$, while at $r>r_{j 1} X=0.7$, and $Z=0.02$. Besides, $r_{11} / R=0.235$, and $r_{\mathrm{u}} / R=0.789$. 


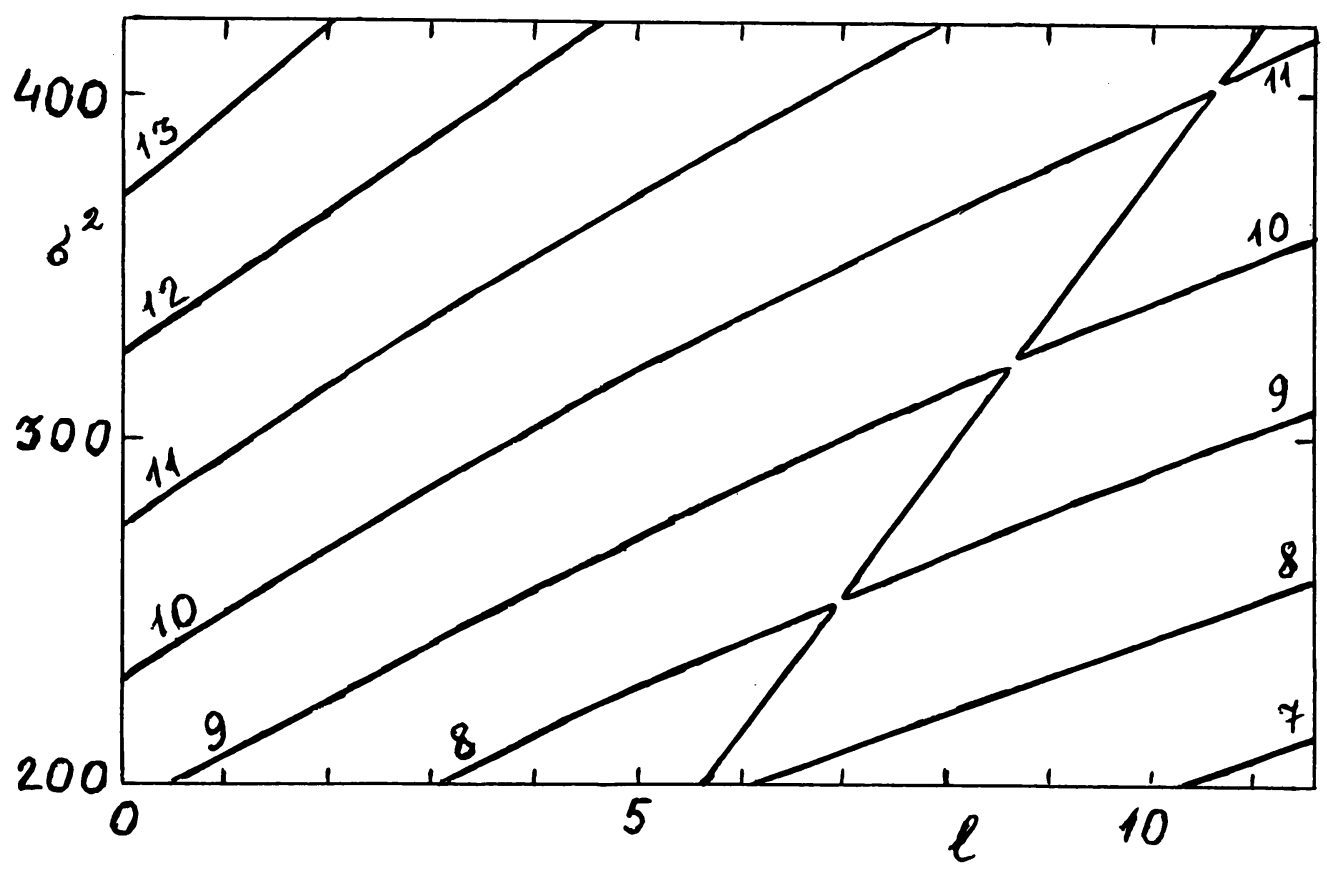

Figure 1.

Figure 1 illustrates the unusual character of the phenomenon of avoided crossing. Here a part of the diagram $\sigma^{2}-\ell$ is shown where $\sigma^{2}=R^{3} \omega^{2} /(G M)$ is nondimensional frequency squared. The numbers of radial nodes are shown near the curves which join the $\sigma^{2}$ corresponding to different $\ell$-values. The frequencies of the interior modes lie on the nearly straight line tending to cross the $\sigma^{2}$ of p-modes. Note that the interior modes are unstable if $5 \leq \ell \leq 9$ with the time of growth of the order of the age of the Sun.

Of course, the interior modes have very small amplitudes near the surface. Nevertheless, one may hope that in their presence, some observable phenomena on the surface can occur due to interactions of the interior modes with magnetic fields or due to some resonance mode coupling.

\section{REFERENCES}

Gabriel, M., Scuflaire, R., 1979: Acta Astron., 29, 135.

Rouse, C.A., 1983: Astron. Astrophys., 126, 102.

Spruit, H.C., 1974: Solar Phys., 34, 277.

Vandakurov, Yu.V.: 1984a, Astron. Zh. 61, 170.

Vandakurov, Yu.V.: 1984b, Pis'ma Astron. Zh. 10, 873. 